\title{
State Feedback Set Stabilization for a Class of Nonlinear Systems
}

\author{
Lars Imsland and Bjarne A. Foss \\ Department of Engineering Cybernetics, Norwegian Univ. of Science and \\ Technology, 7491 Trondheim, Norway \\ \{Lars.Imsland, Bjarne.A.Foss\}@itk.ntnu.no
}

\begin{abstract}
A controller for a class of multiple input systems is proposed, with a subset of nonlinear positive systems as a special case. The controller is shown to obtain set stability of a certain subset of the state space. A simple example illustrates the theory.
\end{abstract}

\section{Introduction}

The class of nonlinear systems is very diverse, and encompasses many types of applications and behaviors. It seems apparent that for control purposes, one should focus on specific nonlinear system classes to exploit the structure of these, rather than make an all-encompassing theory for nonlinear systems.

One class of nonlinear systems that has strong structural constraints that can be exploited, is the class of positive systems. The typical class of nonlinear positive systems seems to be systems based on material balances, for example compartmental systems (Jacquez and Simon, 1993) and systems based on mass balances (Bastin, 1999). These system classes enjoy in addition to positivity structural constraints related to the flow of "mass" between the states. Similar structural constraints are assumed on the system class in Imsland and Foss (2002), Imsland (2002).

In this paper, the situation compared to Imsland and Foss (2002) is generalized. As an outset, we consider a (non-positive) system which have a set of positive inventories (which can be interpreted as mass, energy, entropy, ...). This allows more general processes than mass balance systems. Furthermore, the structural constraints are related to a general nonlinear function (rather than the sum) of the states, and the controller aims at a constant value of this nonlinear function. Hence, the "total mass"-approach used in Imsland and Foss (2002) becomes a special case of the approach used herein. Similarly to Imsland and Foss (2002) we allow multiple-input systems by dividing the states into what we call phases. 
The considered system class is presented in Section 2, while we in Section 3 first recapitulates the necessary Lyapunov theorems before we give the main result (Theorem 3). After a brief discussion in Section 4, we present a simple example to illustrate the results.

The notation is fairly standard. $|z|_{\mathcal{A}}$ denotes the distance from $z$ to the set $\mathcal{A}$, defined by $|z|_{\mathcal{A}}:=\inf _{\tilde{z} \in \mathcal{A}}\|z-\tilde{z}\|$ where $\|\cdot\|$ is the Euclidean norm. Furthermore, $\mathbb{R}_{+}=[0, \infty)$.

\section{System class}

Consider the input affine state-space system

$$
\dot{x}=f(x)+g(x) u,
$$

where $x \in \mathbb{R}^{n}$ and $u \in \mathbb{R}_{+}^{m}$. The inputs are positive and governs in some sense the inflow of the process system.

For this system, there exists of a positive vector function of "inventories" (Farsham, Viswanath and Ydstie, 1998) $\nu(x)=\left(\nu_{1}(x), \ldots, \nu_{p}(x)\right) \subset \mathbb{R}_{+}^{p}$, which behave according to

$$
\dot{\nu}=\frac{\partial \nu}{\partial x} f(x)+\frac{\partial \nu}{\partial x} g(x) u:=F(x)+G(x) u .
$$

At this point, note that the positive system $\dot{\nu}=F(\nu)+G(\nu) u$ is a special case.

We will assume that the inventories are divided into $m$ different groups (one for each input), which for convenience will be denoted phases. Phase $j$ will consist of $r_{j}$ inventories (with $\sum_{j=1}^{m} r_{j}=p$ ), and have the control $u_{j}$ associated with it (controlling the inflow to that phase). The inventories in phase $j$ will be denoted $\nu^{j}$, such that $\nu=\left(\nu^{1}, \ldots, \nu^{m}\right)$. Note that $m \leq p$, such that $\nu^{j}$ of phase $j$ can consist of several inventories $\nu_{i}$. Corresponding to this structure, we write the vector function $F(x)$ and the matrix function $G(x)$ as

$$
\begin{aligned}
& F(x)=\left(F^{1}(x), \ldots, F^{m}(x)\right), \quad F^{j}(x)=\left(\phi_{1}^{j}(x), \ldots, \phi_{r_{j}}^{j}(x)\right), \\
& G(x)=\operatorname{blockdiag}\left(G^{1}(x), \ldots, G^{m}(x)\right), \quad G^{j}(x)=\left(\gamma_{1}^{j}(x), \ldots, \gamma_{r_{j}}^{j}(x)\right) .
\end{aligned}
$$

For each phase, we will choose a positive scalar function $H_{j}\left(\nu^{j}\right)$ such that the control objective is that the inventories of phase $j$ should converge to the "level set" $\Omega_{j}$, given by $\Omega_{j}:=\left\{z \in \mathbb{R}^{r_{j}} \mid H_{j}(z)=C_{j}\right\}$. The inventories can typically be interpreted as mass, or energy, and the functions $H_{j}\left(\nu^{j}\right)$ as total mass or energy (of a "subprocess"). In some sense, the functions $H_{j}\left(\nu^{j}\right)$ are also inventories, but we distinguish these since the control objective is related to them.

Define the scalar function $V_{j}(z):=\frac{1}{2}\left(H_{j}(z)-C_{j}\right)^{2}$, on which we impose 
Assumption 1 The sets $\Omega_{j}$ are compact, and there exist constants $\underline{c}^{j}<\bar{c}^{j}$ such that for all $z \in \mathbb{R}^{r_{j}}$,

$$
\underline{c}^{j}|z|_{\Omega_{j}} \leq V_{j}(z) \leq \bar{c}^{j}|z|_{\Omega_{j}} .
$$

Remark 1. The above assumption holds (see Imsland (2002)) for example if we for a positive system (with states $\nu$ ) choose the "total mass" $H_{j}\left(\nu^{j}\right)=$ $\sum_{i=1}^{r_{j}} \nu_{i}^{j}$, which is used e.g. in Bastin and Praly (1999) and Imsland and Foss (2002). The positivity of the system $\left(\nu^{j} \in \mathbb{R}_{+}^{r_{j}}\right)$ ensures in this case that $\Omega_{j}$ is compact, which is essential.

The system assumptions are made with respect to the set $D \subseteq \mathbb{R}^{n}$ :

Assumption 2 The function $F(x): D \rightarrow \mathbb{R}^{n}$ is locally Lipschitz and for $x \in\left\{x \in D \mid H_{j}\left(\nu^{j}(x)\right)>C_{j}\right\}, \sum_{i=1}^{r_{j}} \frac{\partial H_{j}\left(\nu^{j}\right)}{\partial \nu_{i}^{j}} \phi_{i}^{j}(x) \leq 0$ and the set $\left\{x \in D \mid \sum_{i=1}^{r_{j}} \frac{\partial H_{j}\left(\nu^{j}\right)}{\partial \nu_{i}^{j}} \phi_{i}^{j}(x)=0\right.$ and $\left.H_{j}\left(\nu^{j}\right)>C_{j}\right\}$ does not contain an invariant set.

This assumption means that $F(x)$ consists of interconnection terms (terms that have zero net contribution to $H_{j}\left(\nu^{j}\right)$, that is, "flow" between different inventories in a phase), and the rest of the terms are dominantly outflow (at least for large $H_{j}\left(\nu^{j}\right)$ ).

Assumption 3 The block diagonal matrix function $G(x): D \rightarrow \mathbb{R}^{n \times m}$ is locally Lipschitz and $\sum_{i=1}^{r_{j}} \frac{\partial H_{j}}{\partial \nu_{i}^{j}} \gamma_{i}^{j}(x)>0$ for all $x \in D$.

Note that further assumptions are needed to ensure that the inventories remain positive, but these are implicitly required to hold.

\section{State feedback set stabilization}

A Lyapunov condition for asymptotic stability of a compact set $\mathcal{A}$ is:

Theorem 1. The compact set $\mathcal{A}$ is asymptotically stable (in the sense of Lyapunov) for $\dot{x}=f(x)$ if there on an open set $\mathcal{O}$ containing $\mathcal{A}$ exists a $C^{1}$ function $V$ and positive constants $\kappa_{1}$ and $\kappa_{2}$ such that

$$
\begin{aligned}
\kappa_{1}|x|_{\mathcal{A}} & \leq V(x) \leq \kappa_{2}|x|_{\mathcal{A}} \\
\frac{\partial V}{\partial x} f(x) & <0 \text { for }|x|_{\mathcal{A}} \neq 0
\end{aligned}
$$

on the set $\mathcal{O}$.

See e.g. Rouche, Habets and Laloy (1977) for a treatment of set stability. The above condition is not the most general, but sufficient for our purpose.

In some cases, it is hard to find a Lyapunov function where the derivative is negative definite. If it is semidefinite, a straightforward adoption of LaSalle's invariance principle (similar to the Barabashin-Krasovskii Theorem) can be used: 
Theorem 2. If the conditions of Theorem 1 hold on the compact set $\mathcal{D}$ (containing $\mathcal{A}$ ) except that (5) is replaced with

$$
\frac{\partial V}{\partial x} f(x) \leq 0
$$

on $\mathcal{D}$, and the largest invariant set contained in the set where $\frac{\partial V}{\partial x} f(x)=0$ is contained in $\mathcal{A}$, then the set $\mathcal{A}$ is asymptotically stable for $\dot{x}=f(x)$.

Recalling that asymptotic stability is equivalent to stability and convergence (Rouche et al., 1977), this result is obtained by noting that the semidefiniteness of the derivative of the Lyapunov function implies (set) stability, while convergence follows from LaSalle's invariance principle (LaSalle, 1960).

The controller we suggest for the given system class, is

$u_{j}(x)=\max \left\{0,\left[\sum_{i=1}^{r_{j}} \frac{\partial H_{j}}{\partial \nu_{i}^{j}} \gamma_{i}^{j}(x)\right]^{-1}\left(-\sum_{i=1}^{r_{j}} \frac{\partial H_{j}}{\partial \nu_{i}^{j}} \phi_{i}^{j}(x)-\lambda_{j}\left(H_{j}\left(\nu^{j}\right)-C_{j}\right)\right)\right\}$

where the $\lambda_{j}$ s are positive constants. Defining $\Omega:=\bigcup_{j=1}^{m} \Omega_{j}$, we make the following assumption:

Assumption 4 There exists a (not necessarily compact) set $D$ that is i) invariant for the dynamics (1) in closed loop with control (7), ii) has a nonempty intersection with $\Omega$, and iii) for $x \in \Omega \cap D, u_{j}(x)>0$.

The stability properties can be summarized as follows:

Theorem 3. Under Assumptions 1-4, the set $\Omega \cap D$ is asymptotically stable for the closed loop system controlled with (7). Moreover, convergence to $\Omega \cap D$ and boundedness of trajectories holds for initial conditions $x(0) \in D$.

Proof. The set $D$ is by Assumption 4 invariant, hence Assumptions 2 and 3 hold along closed loop trajectories. By Assumption 3, the control (7) is well defined on $D$.

Define the positive semidefinite function $V(x):=\sum_{j=1}^{m} V_{j}\left(\nu^{j}(x)\right)$, with time derivative along system trajectories

$$
\begin{aligned}
\dot{V}(x) & =\sum_{j=1}^{m}\left[H_{j}\left(\nu^{j}(x)\right)-C_{j}\right] \dot{H}_{j}\left(\nu^{j}(x)\right) \\
& =\sum_{j=1}^{m}\left[H_{j}\left(\nu^{j}(x)\right)-C_{j}\right]\left(\sum_{i=1}^{r_{j}} \frac{\partial H_{j}}{\partial \nu_{i}^{j}} \phi_{j}^{i}(x)+\sum_{i=1}^{r_{j}} \frac{\partial H_{j}}{\partial \nu_{i}^{j}} \gamma_{j}^{i}(x) u_{j}(x)\right) .
\end{aligned}
$$

For $H_{j}\left(\nu^{j}\right) \neq C_{j}$, we have one of the following cases:

1. If $u_{j}(x)>0$, summand $j$ is

$$
\left[H_{j}\left(\nu^{j}\right)-C_{j}\right]\left(\sum_{i=1}^{r_{j}} \frac{\partial H_{j}}{\partial \nu_{i}^{j}} \phi_{j}^{i}(x)+\sum_{i=1}^{r_{j}} \frac{\partial H_{j}}{\partial \nu_{i}^{j}} \gamma_{j}^{i}(x) u_{j}(x)\right)=-\lambda_{j}\left[H_{j}\left(\nu^{j}\right)-C_{j}\right]^{2}<0 .
$$


2. If $u_{j}(x)=0$, then summand $j$ is $\left[H_{j}\left(\nu^{j}\right)-C_{j}\right] \sum_{i=1}^{r_{j}} \frac{\partial H_{j}}{\partial \nu_{i}^{j}} \phi_{j}^{i}(x)$. Thus, if $H_{j}\left(\nu^{j}\right)>C_{j}$, then by Assumption $2 \sum_{i=1}^{r_{j}} \frac{\partial H_{j}}{\partial \nu_{i}^{j}} \phi_{j}^{i}(x) \leq 0$, and

$$
\left[H_{j}\left(\nu^{j}\right)-C_{j}\right] \sum_{i=1}^{r_{j}} \frac{\partial H_{j}}{\partial \nu_{i}^{j}} \psi_{j}^{i}(x) \leq 0 .
$$

If $H_{j}\left(\nu^{j}\right)<C_{j}$, then since $u_{j}(x)=0$ and $\sum_{i=1}^{r_{j}} \frac{\partial H_{j}}{\partial \nu_{i}^{j}}>0$ by Assumption 3, we see that $\sum_{i=1}^{r_{j}} \frac{\partial H_{j}}{\partial \nu_{i}^{j}} \phi_{j}^{i}(x) \geq-\lambda_{j}\left[H_{j}\left(\nu^{j}\right)-C_{j}\right]$, which gives

$$
\left[H_{j}\left(\nu^{j}\right)-C_{j}\right] \sum_{i=1}^{r_{j}} \frac{\partial H_{j}}{\partial \nu_{i}^{j}} \phi_{j}^{i}(x) \leq-\lambda_{j}\left[H_{j}\left(\nu^{j}\right)-C_{j}\right]^{2}<0 .
$$

If $H_{j}\left(\nu^{j}\right)=C_{j}, \nu^{j} \in \Omega_{j}$ and $\dot{V}_{j} \equiv 0$ due to Assumption 4 iii).

This holds for all summands (phases), and we conclude that $\dot{V}(x) \leq 0$. This implies that $V(x(t)) \leq V\left(x\left(t_{0}\right)\right)$ along system trajectories. From the construction of $V$ and Assumption 1, we see that $\|x\| \rightarrow \infty$ if and only if $V(x) \rightarrow \infty$, hence bounded $V(x(t))$ implies that $\|x(t)\|$ is bounded (stays in the compact set given by $\left.\left\{x \mid V(x) \leq V\left(x\left(t_{0}\right)\right)\right\}\right)$.

We conclude by Theorem 2 that $\Omega \cap D$ is asymptotically stable, since by Assumption 1, $V$ fulfills (4) with respect to $\Omega$, and as shown above, $\dot{V}(x) \leq 0$ for $x \in D$. Moreover, from the above and Assumption 2, the only invariant set where $\dot{V}(x)=0$ is (within) $\Omega \cap D$.

Remark 2. For positive systems, the set $D$ can in many cases be taken as the positive orthant, $D=\mathbb{R}_{+}^{n}$. Another option can be "Lyapunov level sets" of the type

$$
\left\{x \in \mathbb{R}^{n} \mid C_{j}-\underline{\beta}_{j} \leq H_{j}\left(\nu^{j}(x)\right) \leq C_{j}+\bar{\beta}_{j}, j=1, \ldots, m\right\} .
$$

Similarly to De Leenheer and Aeyels (2002), we can state the following result on asymptotic stability of equilibria. It can be proved the same way as in De Leenheer and Aeyels (2002), or seen as a consequence of the theory of semidefinite Lyapunov functions (Chabour and Kalitine, 2002), hence we state it without proof:

Theorem 4. Let the conditions of Theorem 3 hold. If the closed loop (1) with control (7) has a single equilibrium in the interior of $\Omega \cap D$ that is asymptotically stable with respect to initial conditions in $\Omega \cap D$ and attractive for all initial conditions in $\Omega \cap D$, the equilibrium is asymptotically stable for the closed loop with a region attraction (of at least) D. 


\section{Discussion of controller}

\subsection{Connection with similar control schemes}

The trained eye will see that the proposed controller is similar to feedback linearizing controllers (as, for instance, in Isidori (1995)). However, while feedback linearization linearizes the whole state space, (7) linearizes (in the unconstrained case) only the "phase dynamics" (the dynamics of the functions $H_{j}\left(\nu^{j}(x)\right)$ ), while the total dynamics remain nonlinear. Feedback linearization requires in general solving a set of PDEs to find the right "full relative degree" output, while for the approach herein, the functions $H_{j}$ are given by assumption. Furthermore, the present approach can preserve stability under some input constraints, due to the system properties.

The controller (7) is the same as proposed in Bastin and Praly (1999) (see also Bastin and Provost (2002)). Herein, a larger class of systems is studied, for instance the concept of phases allow to handle systems with multiple inputs. This idea is also explored in Imsland and Foss (2002), Imsland (2002) in the setting of positive systems, but herein the concept of "inventories" allows us to move beyond positive systems. Moreover, the approach in Imsland and Foss (2002) is generalized herein in allowing more general functions $H_{j}$.

The inventory concept is borrowed from Farsham et al. (1998), where interesting connections are tied between the first and second law of thermodynamics and system theoretic properties of "process systems". The control problem therein is to steer the inventories to their setpoints, and this is done by assuming it is possible to manipulate the flows corresponding to each inventory. Only convergence of the inventories are considered, the underlying system behavior is not examined. One major difference to the controller developed herein, is that we do not assume a one-to-one relationship between inventories and manipulated flows.

Constraints are considered in Farsham et al. (1998) by augmenting the controller to a PID type controller with antireset windup. The "original" controller is in this setting a $\mathrm{P}$-controller, in the same way as the one proposed herein (interpreting the $\lambda_{j}$ s as gains).

\subsection{Outflow controlled systems and upper constraints}

In view of Assumption 3 (and the sign of $H_{j}$ ), it can be said that the system class treated herein is inflow controlled (with respect to $H_{j}$ ). However, systems that in a corresponding sense are outflow controlled (or having a mixture of inflow or outflow controlled phases), can be treated in a similar way. This is done in Imsland and Foss (2002) for positive systems with $H_{j}\left(\nu^{j}\right)=\sum_{i=1}^{r_{j}} \nu_{i}^{j}$. In addition to more rigid conditions on the "uncontrolled" flows, one must then in general have an upper saturation on the control to ensure that the inventories remain positive. 


\section{Example}

For mass balance systems where $H_{j}\left(\nu^{j}\right)$ is the sum of the phase states (masses), $H_{j}\left(\nu^{j}\right)=\sum_{i=1}^{r_{j}} \nu_{i}^{j}$, several examples can be found in Imsland (2002), for instance the stabilization of a gas lifted oil well (Imsland and Foss, 2002).

Here we will consider an example which is a (slightly modified) system taken from De Leenheer and Aeyels (2002), which illustrate another type of $H_{j}$ for a positive system (states are inventories). The system equations are

$$
\begin{aligned}
& \dot{x}_{1}=-x_{1} x_{2}+x_{2}^{2}-x_{1}+u \\
& \dot{x}_{2}=-x_{1} x_{2}+x_{1}^{2}+u .
\end{aligned}
$$

We choose $H(x)=\frac{1}{2}\left(x_{2}^{2}+x_{2}^{2}\right)$, which gives $\frac{\partial H}{\partial x} F(x)=-x_{2}^{2}$ and $\frac{\partial H}{\partial x} G(x)=$ $x_{1}+x_{2}$. The assumptions $2-3$ hold on $D=\mathbb{R}_{+}^{2}$, hence by Theorem 3 the control law given by (7) globally (on the positive orthant) stabilizes the set $\frac{1}{2}\left(x_{1}^{2}+x_{2}^{2}\right)=C$, for any positive $C$.

Furthermore, it is rather easy to show that on the set $H(x)=C$ the system has a single equilibrium at $x_{1}^{\star}=\frac{\sqrt{1+16 C}-1}{4}, x_{2}^{\star}=\sqrt{2 C-\left(x_{1}^{\star}\right)^{2}}$ which is asymptotically stable with respect to the set $H(x)=C$. By Theorem 4, this equilibrium is stable for all initial conditions in $\mathbb{R}_{+}^{2}$.

A simulation confirming these results is shown in Figure 1. Note the initial input saturation.
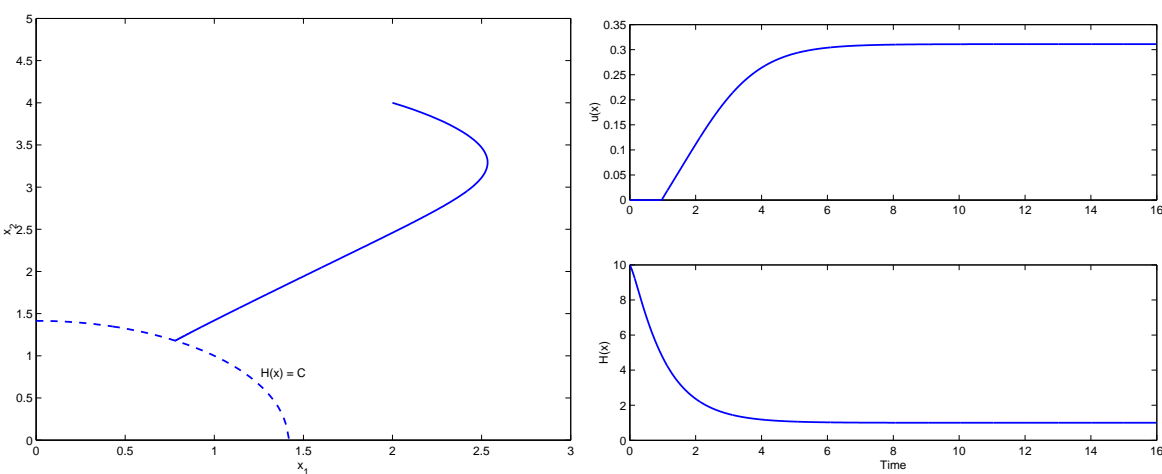

Fig. 1. To the left, a phase-plot, to the right $u(x)$ and $H(x)$. The simulation had initial condition $(2,4)$, and the parameters $C=1$ and $\lambda=1$.

\section{Concluding remarks}

The concept of set stability is used to demonstrate the stability properties of a certain positive controller, for a special system class. One important restriction of the system class is the assumptions that ensure that the "Lyapunov 
function" used in the proof of the main result is decreasing when the input saturates. According to Bastin and Praly (1999), this condition can be seen as a dissipativity property of the system.

\section{Acknowledgment}

The NTNU Natural Gas Research Center is acknowledged for financial support.

\section{References}

Bastin, G. (1999). Issues in modelling and control of mass balance systems, in D. Aeyels, F. Lamnabi-Lagarrigue and A. van der Schaft (eds), Stability and stabilization of Nonlinear Systems, Springer.

Bastin, G. and Praly, L. (1999). Feedback stabilisation with positive control of a class of dissipative mass-balance systems, Proceedings of the 14th IFAC World Congress, Beijing, P. R. China.

Bastin, G. and Provost, A. (2002). Feedback stabilisation with positive control of dissipative compartmental systems, Proceedings of MTNS.

Chabour, R. and Kalitine, B. (2002). Semi-definite Lyapunov functions stability and stabilizability, IEEE Trans. Aut. Control. To appear.

De Leenheer, P. and Aeyels, D. (2002). Stabilization of positive systems with first integrals, Automatica 38(9): 1583-1589.

Farsham, C. A., Viswanath, K. P. and Ydstie, B. E. (1998). Process systems and inventory control, AIChE Journal 44(8): 1841-1857.

Imsland, L. and Foss, B. A. (2002). A state feedback controller for a class of positive systems: Application to gas lift stabilization, Submitted to European Control Conference, 2003, Cambridge, England.

Imsland, L. S. (2002). Topics in Nonlinear Control - Output Feedback Stabilization and Control of Positive Systems, PhD thesis, Norwegian University of Science and Technology, Department of Engineering Cybernetics.

Isidori, A. (1995). Nonlinear control systems, third edn, Springer-Verlag, Berlin.

Jacquez, J. A. and Simon, C. P. (1993). Qualitative theory of compartmental systems, SIAM Rev. 35(1): 43-79.

LaSalle, J. P. (1960). Some extensions of Liapunov's second method, IRE Trans. CT-7: 520-527.

Rouche, N., Habets, P. and Laloy, M. (1977). Stability theory by Liapunov's direct method, Springer-Verlag, New York. Applied Mathematical Sciences, Vol. 22. 